\title{
An international comparison of surface texture parameters quantification on polymer artefacts using optical instruments
}

\author{
G. Tosello (2) ${ }^{1}$, H. Haitjema (2) ${ }^{2}$, R. K. Leach ${ }^{3}$, D. Quagliotti ${ }^{1}$, S. Gasparin $(3)^{4}$, H. N. Hansen (1) ${ }^{1}$ \\ ${ }^{1}$ Department of Mechanical Engineering, Technical University of Denmark, Building 427A, Produktionstorvet, 2800, Kgs. Lyngby, Denmark \\ ${ }^{2}$ Mitutoyo Research Center Europe B.V., De Rijn 18, 5684 PJ Best, The Netherlands \\ ${ }^{3}$ Faculty of Engineering, University of Nottingham, B92, Coates Building, University Park, Nottingham, NG7 2RD, UK \\ ${ }^{4}$ LEGO System A/S, Åstvej, 7190 Billund, Denmark
}

An international comparison of optical instruments measuring polymer surfaces with arithmetic mean height values in the sub-micrometre range has been carried out. The comparison involved sixteen optical surface texture instruments (focus variation instruments, confocal microscopes and coherent scanning interferometers) from thirteen research laboratories worldwide. Results demonstrated that: (i) agreement among different instruments could be achieved to a limited extent; (ii) standardized guidelines for uncertainty evaluation of areal surface parameters are needed for users; (iii) it is essential that the performance characteristics (and especially the spatial frequency response) of an instrument is understood prior to a measurement.

Optical, Surface, Comparison

\section{Introduction}

Advanced precision manufacturing processes are capable of producing surfaces with structured micro- and nano-scale topographic features (e.g., by replication [1]), to achieve specific functional properties (e.g., optical, fluidic, etc.) [2]. Such structured surfaces require areal surface texture measurements for their characterisation. Optical instruments are widely used to characterise areal topographies. Compared to mechanical probe instruments, optical instruments perform measurements much faster without damaging the surface.

A measurement traceability and performance verification framework for optical instruments measuring areal surface texture parameters is needed to support the quality control of surface manufacturing [3]. In order to investigate the state-ofthe-art in this area, an international comparison of optical instruments measuring polymer surfaces with sub-micrometre texture scales was carried out in the period from 2013 to 2015.

The comparison involved sixteen optical surface instruments (three focus variation microscopes (FVs), four confocal microscopes (CFs) and nine coherence scanning interferometers (CSIs)) from thirteen research laboratories in ten countries worldwide. The comparison was organised by the Scientific Technical Committee 'Surfaces' of the International Academy for Production Engineering (CIRP).

A number of metrological comparisons (see e.g., [4-9]) have been carried out on surfaces on the micro- to the nano-scale, measuring profile surface texture parameters $[5,8]$ and step heights $[4,6,7]$, using either contact instruments or scanning probe microscopes. In references $[8,9]$, non-contact methods (optical) were also used but the investigation was still limited to profile roughness parameters.

Compared to previous comparisons, the present work focuses for the first time on areal surface texture parameters acquired by optical instruments on polymer surfaces. The aim was to identify the current status of the measuring capability for areal surface texture in laboratory conditions, with knowledgeable operators.

\section{Description of the transfer standards}

A set of four nickel master transfer standards, manufactured by Rubert + Co. Ltd., UK [10], were replicated by injection moulding (see Figure 1 (a)).

Two nickel transfer standards, with serial numbers 528 and 529 , were of ISO type C with nominal periodic profile of $R a=500 \mathrm{~nm} \quad(R S m=50 \mu \mathrm{m})$ and $R a=100 \mathrm{~nm} \quad(R S m=10 \mu \mathrm{m})$ respectively, and two were of ISO type $\mathrm{D}$, with serial numbers 502 and 503, with random profiles of nominally $R a=30 \mathrm{~nm}$ and $R a=100 \mathrm{~nm}$ respectively. A detailed description of profile ISO transfer standard types can be found elsewhere [11].

The polymer replicas were eighteen sets of transfer standards, injection moulded using a commercially available ABS (acrylonitrile butadiene styrene), Cycolac KJY 039075 by Borg Warner, with a grey colour (see an example in Figure 1 (b)). Injection mouldings have been carried out on an injection moulding machine with a reciprocating screw of $35 \mathrm{~mm}$ in diameter and a clamping force of $600 \mathrm{kN}$. Moulding process parameters, such as a melt temperature of $230^{\circ} \mathrm{C}$, mould temperature of $50{ }^{\circ} \mathrm{C}$, injection speed of $50 \mathrm{~mm} \mathrm{~s}^{-1}$, have been used. A total cycle time of about $60 \mathrm{~s}$ was achieved during production. The eighteen sets of specimens were produced in the same batch in controlled and repeatable processing conditions.

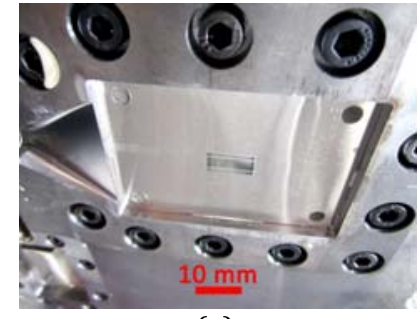

(a)

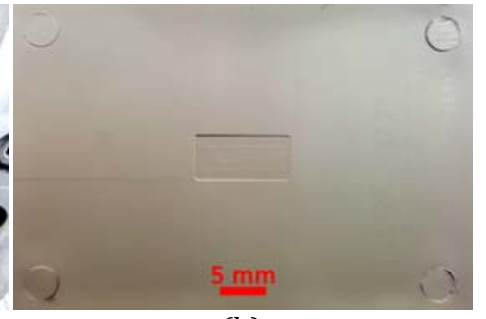

(b)
Figure 1. (a) Example of Rubert's nickel transfer standards used as mould insert; (b) example of polymer replica. 


\section{Measurement procedure}

The replication of the transfer standards by polymer moulding allowed several laboratories to measure in parallel, in contrast to comparisons where a single set of specimens has to be transferred between laboratories. The measurements on the polymer transfer standards were analysed over a $250 \mu \mathrm{m} \times 250 \mu \mathrm{m}$ evaluation area from which a least-squares plane was removed. Arithmetical mean height of the scale-limited surface $(S a)$, root mean square height of the scale-limited surface $(S q)$ and root mean square gradient of the scale-limited surface $(S d q)$ areal texture parameters [12] were calculated and reported. A Gaussian S-filter [13] was applied (with no L-filter). When comparing measurements it is essential that the spatial bandwidths match. This was achieved by having all the measurements taken on the same area, with the same number of points and with the same filter settings [14]. Any variation from the recommended procedure was documented. The $2.5 \mu \mathrm{m}$ nesting index S-filter was applied to reduce variation due to different numbers of points and/or the different lateral resolutions of the instruments. An L-shaped reference mark, engraved in the centre of each nickel standard, was replicated in the polymer sample (see Figure $1(\mathrm{~b})$ ) and used as a local reference system to be aligned with the $x$ and $y$ axes of the instrument. The acquisition area was identified with its leftbottom corner at a distance of $1 \mathrm{~mm}$ in both positive $x$ and $y$ directions from the origin (corner of the mark) of the local reference system.

\section{Reference measurements}

The replicated polymer standards provided to the laboratories were measured before the start of the comparison using an atomic force microscope (AFM). These measurements were treated as the reference values to which all the measurements were referenced. The uncertainty for the AFM measurements was quantified using a procedure inspired by an international standard focused on coordinate measuring machines uncertainty assessment [15]. The following influence factors were considered in the uncertainty assessment: background noise of the AFM; uncertainty from the calibration artefact certificate; measurement repeatability (including instrument, relocation and workpiece); and calibration uncertainty of the instrument.

\section{Analysis of participants' data}

The analysis of the participants' data was carried out using the $E_{n}$ value determination for all instruments and measurands. The relative deviations from the references for all the surface texture parameters were calculated and the uncertainties stated by the participants related to those evaluated for the references.

\section{Table 1}

Uncertainty contributors related to the different instruments involved in the comparison; ( $\square$ ): letters refer to the instruments; ( $\square$ ): FV = focus variation, $\mathrm{CSI}=$ coherence scanning interferometer, $\mathrm{CF}=$ confocal microscope.

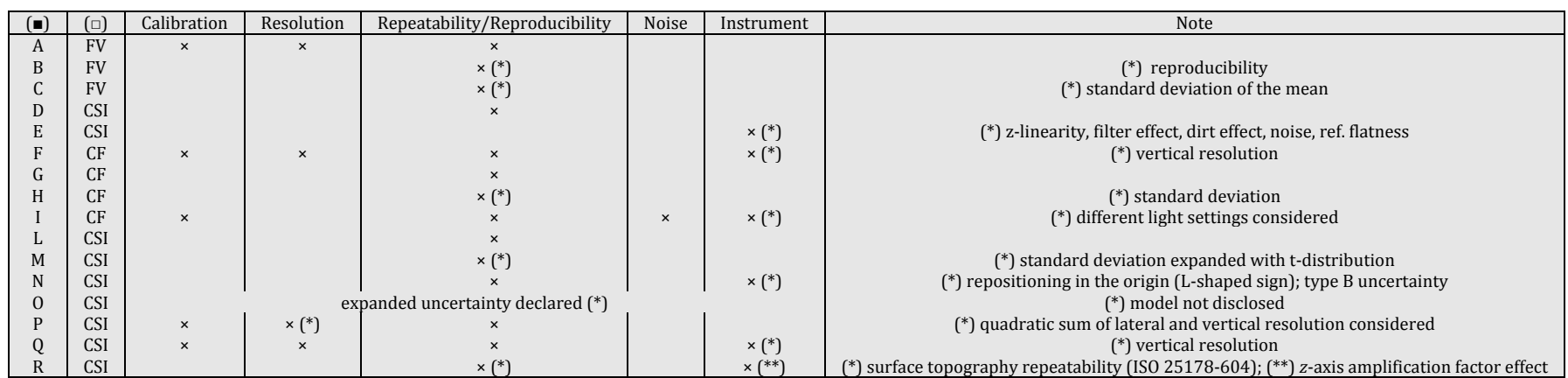

Examples of results of the comparison are shown in Figure 2 and in Figure 3 for the 502 and 529 samples respectively.

\subsection{Uncertainty evaluation}

The participants were asked to evaluate the uncertainty using their own preferred method; the uncertainty quantification was also a subject of the comparison. The uncertainty models employed are categorised in Table 1, according to the influence factors for each instrument that each participant considered relevant. In those cases where the uncertainty evaluation was based on repeatability only, the result was that the deviations between optical measurements and reference values were larger than the uncertainty, indicating the likelihood of an uncertainty underestimation. When a more comprehensive uncertainty model was applied, a more realistic uncertainty value was obtained and agreement was observed in some cases. A further analysis of the uncertainty budget indicated that in some cases calibrated artefacts having a calibration uncertainty of the same order of magnitude as the amplitude of the specimens under investigation were used, resulting in an overestimation of uncertainty.

\section{2. $E_{n}$ value}

The $E_{n}$ value was determined under the assumptions that there was no correlation among the different results and that the participants have used similar methods to assess uncertainty. Hence, the $E_{n}$ determination is expected to indicate (when $\left|E_{n}\right|>1$ ) whether an instrument is affected by unforeseen issues in its measurements and/or uncertainty evaluations.

$E_{n}$ has been calculated according to the following equation:

$$
E_{n}=\frac{S x_{j}-S x_{A F M}}{\sqrt{U\left(S x_{j}\right)^{2}+U\left(S x_{A F M}\right)^{2}}}
$$

where: $\mathrm{Sx}_{j}=S a, S q, S d q$ results obtained with the $j$-th optical instrument; $S X_{A F M}=$ corresponding reference value obtained by the AFM; $U\left(S x_{j}\right)=$ uncertainty of the $S x$ result from the $j$-th optical instrument; $U\left(S x_{A F M}\right)=$ uncertainty of the $S x$ result from the AFM. The average values over eighteen specimens and the expanded uncertainties are reported in Table 2. In summary, from the $E_{n}$ analysis the following can be concluded:

Sa: $\quad-8 \%$ of all measurements by FV have $|\mathrm{En}|<1$

- $22 \%$ of all measurements by CSI have $|\mathrm{En}|<1$

- $75 \%$ of all measurements by CF have $\mid$ En $\mid<1$

$S q:$

- $8 \%$ of all measurements by FV have $|\mathrm{En}|<1$

- $19 \%$ of all measurements by CSI have $|\mathrm{En}|<1$

- $63 \%$ of all measurements by CF have $|\mathrm{En}|<1$

Sdq: $\quad-17 \%$ of all measurements by FV have $\left|E_{n}\right|<1$

- $33 \%$ of all measurements by CSI have $\left|E_{n}\right|<1$

- $50 \%$ of all measurements by CF have $\left|E_{n}\right|<1$ 

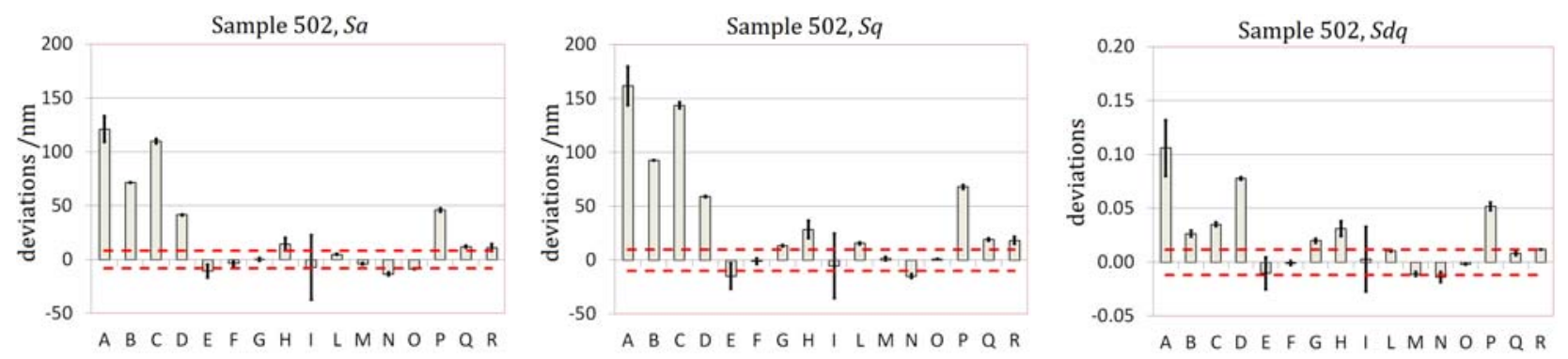

Figure 2. Left: $S a$ deviations for 502 type samples (random profile), expressed in nm. Centre: $S q$ deviations for 502 type samples, expressed in nm. Right: $S d q$ deviations for 502 type samples, dimensionless. Red dashed lines (- - ) indicate the expanded uncertainty of AFM reference measurements. Bars on histograms (
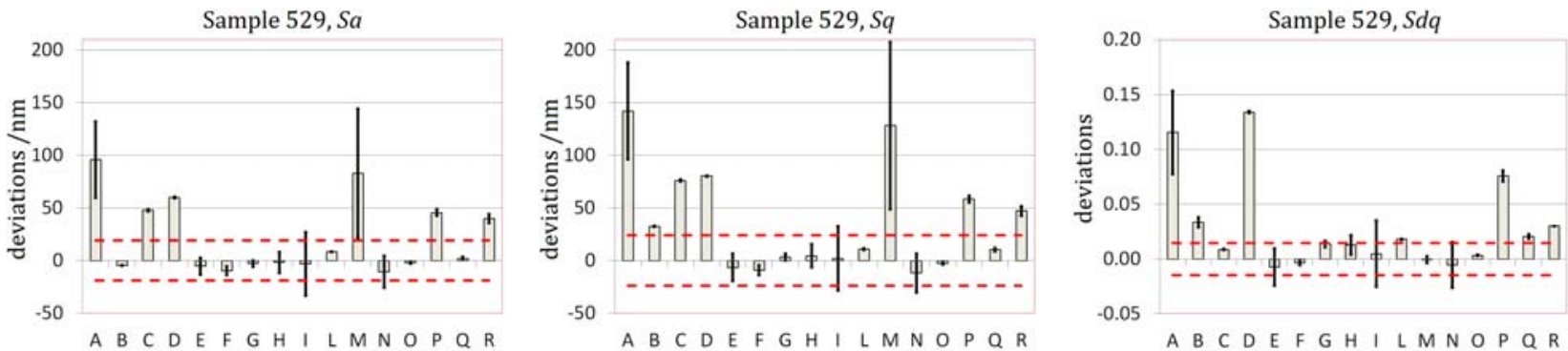

Figure 3. Left: $S a$ deviations for 529 type samples (periodic profile), expressed in nm. Centre: $S q$ deviations for 529 type samples, expressed in nm. Right: $S d q$ deviations for 529 type samples, dimensionless. Red dashed lines (- - ) indicate the expanded uncertainty of AFM reference measurements. Bars on histograms ( $\mathbf{1})$ indicate the expanded uncertainty of the measurements stated by the participants.

Table 2

Average values, standard deviations between specimens and expanded uncertainties of reference AFM measurements calculated over the eighteen replicated polymer samples provided to the laboratories.

\begin{tabular}{|c|c|c|c|c|c|c|c|c|c|c|c|c|}
\hline & \multicolumn{3}{|c|}{502} & \multicolumn{3}{|c|}{503} & \multicolumn{3}{|c|}{528} & \multicolumn{3}{|c|}{529} \\
\hline & $\mathrm{Sa} / \mathrm{nm}$ & $\mathrm{Sq} / \mathrm{nm}$ & $S d q$ & $\mathrm{Sa} / \mathrm{nm}$ & $S q / \mathrm{nm}$ & $S d q$ & $\mathrm{Sa} / \mathrm{nm}$ & $S q / \mathrm{nm}$ & $S d q$ & $\mathrm{Sa} / \mathrm{nm}$ & $S q / \mathrm{nm}$ & $S d q$ \\
\hline Average & 39.0 & 48.8 & 0.044 & 56.3 & 74.3 & 0.073 & 218.8 & 246.3 & 0.051 & 102.6 & 114.1 & 0.078 \\
\hline Std. dev. between specimens & 1.8 & 2.2 & 0.002 & 1.8 & 2.2 & 0.001 & 6.8 & 6.9 & 0.002 & 1.4 & 1.8 & 0.002 \\
\hline Exp. meas. uncertainty ( $\mathrm{k}=2)$ & 3.5 & 4.3 & 0.004 & 3.9 & 4.3 & 0.008 & 13.3 & 15.0 & 0.011 & 3.0 & 4.2 & 0.009 \\
\hline
\end{tabular}

Table 3

Recalculated optical instruments' expanded uncertainties to achieve $\left|E_{n}\right|=0.99$. Average values based on all measurements originally giving $\left|E_{n}\right| \geq 1$.

\begin{tabular}{|c|c|c|c|c|c|c|c|c|c|c|c|c|}
\hline & \multicolumn{3}{|c|}{502} & \multicolumn{3}{|c|}{503} & \multicolumn{3}{|c|}{528} & \multicolumn{3}{|c|}{529} \\
\hline & $U(S a) / \mathrm{nm}$ & $U(S q) / \mathrm{nm}$ & $U(S d q)$ & $U(\mathrm{Sa}) / \mathrm{nm}$ & $U(S q) / \mathrm{nm}$ & $U(S d q)$ & $U(S a) / \mathrm{nm}$ & $U(S q) / \mathrm{nm}$ & $U(S d q)$ & $U(S a) / \mathrm{nm}$ & $U(S q) / \mathrm{nm}$ & $U(S d q)$ \\
\hline $\mathrm{FV}$ & 101.7 & 133.8 & 0.100 & 129.4 & 164.9 & 0.084 & 140.2 & 180.4 & 0.085 & 49.5 & 84.0 & 0.050 \\
\hline CSI & 16.4 & 29.7 & 0.026 & 36.8 & 45.7 & 0.058 & 19.6 & 26.2 & 0.043 & 47.4 & 55.9 & 0.055 \\
\hline $\mathrm{CF}$ & 14.0 & 20.5 & 0.025 & 24.4 & 17.3 & 0.024 & 11.7 & 9.3 & 0.007 & 9.2 & 8.3 & 0.010 \\
\hline
\end{tabular}

A recalculation of the expanded uncertainties in order to achieve $\left|E_{n}\right|=0.99$ was carried out for those results giving $\left|E_{n}\right|>1$. The average values per instrument type are reported in Table 3. By comparing these values with those reported in Table 2, it can be seen that the resulting uncertainties are between one and two orders of magnitude larger than those of the reference values. From this it can be concluded that the results from the optical instruments were affected by high deviations as compared with the reference values in many cases.

\section{Discussion}

A number of observations can be made as a result of the comparison with respect to the different optical instruments, the role of the software handling the data and the surface texture parameters considered. Besides the considerations for each specific instrument, some consistent common trends can be observed and are discussed in the following.

Significant deviations from the AFM reference values for FV instruments results were observed in almost all measured samples. This general issue for the FV instruments indicates that these types of surfaces are not actually suitable to be measured by the FV technology. The main issue is related to the fact that the master and resulting polymer surfaces had local roughness that was too low to allow proper detection by the instrument [16].

Considering the results related to CSI, only a small number of the instruments showed consistent agreement with the corresponding AFM reference values. In general, moderate deviations were observed, sometimes enhanced by unmatched spatial bandwidths. In those cases, it can be observed that the measured area was not exactly as required. In addition, low levels of reflected light and inadequate sensitivity of the detector were observed for some of the measurements.

The raw data acquisitions were commonly affected by noise (spikes and voids), which in some cases, were corrected during the post-processing by the specific instrument software. The correction applied for restoring missing pixels, however, affected the resulting data sets depending on the software, which in turn introduced an additional source of uncertainty, e.g., a loss of information, or even an increased number of spikes. The presence of spikes particularly affected the $S d q$ parameter, which is sensitive to the local slope of the surface. Hence, a robust spike 
reduction process was necessary to obtain consistent final results. Particularly in the case of CF instruments, which had a significant quantity of results in agreement with the AFM reference measurements, it was observed that most of the large deviations were due to the presence of many outliers (i.e. spikes). It was clearly observed that the outlier filters in the image processing software equipped with the instrument used were insufficient in removing the outliers.

Common to all instruments, specific differences related to the $S d q$ parameter were attributed to the local gradients of the polymer surfaces, which exceeded the limits set by the numerical aperture (NA) of the objectives.

\section{Conclusions}

The comparison has highlighted the challenge of measuring areal surface parameters at the $100 \mathrm{~nm}$ scale with optical instruments. Agreement between optical instruments and AFM measurements could be reached to some extent, largely depending on the type of instruments. As a result of the comparison, the following key observations can be made:

- Better performance could be obtained by using CSI and CF microscopes, the latter exhibiting the closest results to the reference values.

- It appeared that these types of surface were not suitable to be measured by FV instruments (due to the effect of local roughness).

- The local gradients of the surface may exceed the limits set by the NA of the objective. This aspect was a key point for CSI, which almost entirely rely on specular reflection.

- Evident noise and spikes were present in several files generated by different optical instruments. Instruments' software showed limitations in removing surface outliers.

- Different spatial bandwidths can make it difficult to compare instruments. At least when comparing results from different instruments, the scanning area and (effective) S-filter must be exactly the same. This is of particular importance when carrying out comparisons and round robin activities.

- Replication of metal transfer standards in polymers proved to be a cost-effective method for production of a high number of transfer standards. The procedure could be applied for future comparisons.

- The differences seen in the comparison exceeded the instruments' specifications in terms of accuracy by several orders of magnitude. Clearly all instruments have their limitations and care must be taken when using them outside the recommended technical specifications.

Furthermore, based on the comparison, the following main topics for further development can be indicated:

- Development of a software package that includes a module for the quantification of the uncertainty of an evaluated surface parameter.

- Development of software tools for a more effective removal of surface outliers.

- Development of appropriate transfer standards for an improved, accurate and comprehensive assessment of the of the instruments uncertainty.

These considerations generated from the direct experience of the participants involved in the comparison are in good agreement with the current state-of-the-art of calibration and verification of areal surface texture measuring instruments [3]. The experience acquired from the present comparison suggests a research roadmap in the field of areal surface texture metrology, which is also in good agreement with the recommendations for future work reported elsewhere [3]. In particular, it is required:

- To complete ISO specification standards (including parts 600 and 700 in the ISO 25178 series).
- To address the lack of primary traceable instruments.

- To conduct formal areal comparisons (such as the one carried out in the present CIRP STC S collaborative activity).

- To establish simplified methods for uncertainty evaluation in industry.

- To develop software measurement standards for all characterisation methods.

- To include fidelity and transfer function work (which still needs an all-encompassing theoretical framework) for an instrument calibration framework.

- To address instrument performance verification (e.g., the framework developed for coordinate measuring systems could be adopted) and possibly its standardisation.

\section{Acknowledgements}

The authors of the current work would like to thank the CIRP colleagues that contributed to the present STC-S collaborative work (in alphabetic order by city name): Dr. O. Riemer and Mr. D. Stöbener (University of Bremen, Germany), Dr. M. Marxer and Prof. C. P. Keferstein (Interstaatliche Hochschule für Technik Buchs, Switzerland), Prof. C. J. Evans (University of North Carolina Charlotte, USA), Prof. B. C. F. Cheung (Hong Kong Polytechnic University, China), Prof. X. Jiang (University of Huddersfield, United Kingdom), Prof. G. Moroni (Politecnico di Milano, Italy), Prof. Enrico Savio (University of Padova, Italy), Prof. K.-D. Bouzakis (University of Thessaloniki, Greece), Prof. F. Z. Fang (University of Tianjin, China), Prof. C. Brown (Worcester Polytechnic Institute, USA), Prof. K. Wegener (Swiss Federal Institute of Technology in Zurich, Switzerland).

\section{References}

[1] Hansen HN, Hocken R, Tosello G (2011) Replication of micro/nano geometries. CIRP Annals - Manufacturing Technology, 60/2:695-714.

[2] Bruzzone A, Costa H, Lonardo P, Lucca DA (2009) Advances in engineered surfaces for functional performance. CIRP Annals - Manufacturing Technology, 57/2:750-769.

[3] Leach RK, Giusca CL, Haitjema H, Evans C, Jiang X (2015) Calibration and verification of areal surface texture measuring instruments. CIRP Annals Manufacturing Technology, 64/2:797-813.

[4] Koenders L, Bergmans R, Garnaes J, Haycocks J, Korolev N, Kurosawa T, Meli F, Park BC, Peng GS, Picotto GB, Prieto E, Gao S, Smereczynska B, Vorburger T, Wilkening G (2003) Comparison on Nanometrology: Nano 2 - Step height Metrologia, Vol. 40 (Technical Supplement)

[5] Doytchinov K, Kornblit F, Colin Castellanos C, Oliveira JCV, Renegar TB, Vorburger TV (2006) International comparison of surface roughness and step height (depth) standards, SIML-S2 (SIM 4.8). Metrologia, Vol. 43 (Technical Supplement).

[6] Koenders L, Klapetek P, Meli F, Picotto GB (2006) Comparison on step height measurements in the nano and micrometre range by scanning force microscopes. Metrologia, Vol. 43 (Technical Supplement).

[7] Danzebrink HU (2009) Supplementary comparison according to the rules of CCL key comparisons - EUROMET project 925 - Intercomparison on Step Height Standards and 1D Gratings - Final Report. Physikalisch-Technische Bundesanstalt.

[8] Baker A, Tan SL, Leach RK, Jung L, Wong SY, Tonmueanwai A, Naoi K, Kim J, Renegar TB, Chaudhary KP, Kruger O, Amer M, Gao S, Tsai CL, Anh N, Drijarkara A (2013) Final report on APMP.L-K8: International comparison of surface roughness. Metrologia, Vol. 50 (Technical Supplement).

[9] Thalmann R (2015) Key Comparison EURAMET.L-K8.2013 - Calibration of surface roughness standards - EURAMET project 1245 - Final report. Metrologia, Vol. 50 (Technical Supplement).

[10] Rubert \& Co Ltd., Reference Specimens, http://www.rubert.co.uk/referencespecimens/ (accessed on January 2016).

[11] ISO 5436 part 1: 2000 Geometrical Product Specification (GPS) - Surface texture: Profile method - Measurements standards - Part 1 Material measures.

[12] ISO 25178 part 2: 2012 Geometrical product specifications (GPS) - Surface texture: Areal - Part 2: Terms, definitions and surface texture parameters.

[13] ISO 25178 part 3: 2012 Geometrical product specifications (GPS) - Surface texture: Areal - Part 3: Specification operators.

[14] Leach RK, Haitjema H (2010) Bandwidth characteristics and comparisons of surface texture measuring instruments. Meas. Sci. Technol. 21:3-9.

[15] ISO 15530-3:2011 Geometrical product specifications (GPS) - Coordinate measuring machines (CMM): Technique for determining the uncertainty of measurement - Part 3: Use of calibrated workpieces or measurement standards.

[16] Helmli F (2011) Focus Variations Instruments. In: Leach RK (Ed.), Optical Measurement of Surface Topography, Springer-Verlag Berlin Heidelberg, Chap. 7 131-166, ISBN 978-3-642-12011-4. 